\title{
Cyclosporine in Patients with Steroid-Resistant Nephrotic Syndrome: An Open-Label, Nonrandomized, Retrospective Study
}

\author{
Gian Marco Ghiggeri, MD, ${ }^{1,2}$ Paolo Catarsi, $\mathrm{PhD},{ }^{2}$ Francesco Scolari, $\mathrm{MD},{ }^{3}$ \\ Gianluca Caridi, PhD, ${ }^{2}$ Roberta Bertelli, PhD, ${ }^{2}$ Alba Carrea, $\mathrm{PhD},{ }^{2}$ \\ Simone Sanna-Cherchi, MD, ${ }^{4}$ Francesco Emma, MD, ${ }^{5}$ Landino Allegri, MD,${ }^{4}$ \\ Giovanni Cancarini, MD, ${ }^{3}$ Gian Franco Rizzoni, MD, ${ }^{5}$ and Francesco Perfumo, $\mathrm{MD}^{1,2}$ \\ ${ }^{1}$ Department of Nephrology and ${ }^{2}$ Laboratory on Pathophysiology of Uremia, G. Gaslini Children's Hospital, Genova, \\ ${ }^{3}$ Nephrology Unit, University of Brescia, Brescia, ${ }^{4}$ Department of Clinical Medicine, Nephrology and Health Sciences, \\ University of Parma, Parma, and ${ }^{5}$ Nephrology Unit, Ospedale Bambin Ges, Rome, Italy
}

\begin{abstract}
Background: Steroid-resistant nephrotic syndrome (SRNS) with focal segmental glomerulosclerosis has emerged as a leading cause of end-stage renal failure (ESRF) in children and adults. In the past decade, immunosuppressive drugs such as cyclosporine (CSA) and cyclophosphamide have been introduced for the treatment of SRNS, but data on long-term clinical outcome (over years) are lacking.

Objective: The current study considered the clinical outcome of patients with SRNS who had been treated with CsA for $>2$ years. The primary objective was to evaluate renal function after years of treatment compared with nontreated or CsA-resistant patients. A secondary objective was to identify renal effects related to the use of CsA, with a major emphasis on renal fibrosis.

Methods: In this open-label, nonrandomized, retrospective study, the outcomes of patients of all ages with sporadic SRNS who had been followed up for $>2$ years (between 1970 and 2002) at 4 Italian clinical institutions were evaluated. Preliminary molecular screenings for genes encoding proteins of the slit-diaphragm (eg, podocin, nephrin, $\alpha$-actinin) were performed to exclude inherited forms of sporadic SRNS.

Results: A total of 157 patients were studied; mutations were found in 18 patients (11\%). Of the remaining 139 patients (84 men, 55 women; median [interquartile range (IQR)] age at onset of proteinuria, 12 [4-32] years), $84(60 \%)$ were nontreated and $55(40 \%)$ were treated with CsA. Of these 55 treated patients, $35(64 \%)$ were found to be resistant (ie, persistence of proteinuria after 2 months) or intolerant (ie, malignant hypertension or worsening of renal function), and CSA was withdrawn. The median (IQR) durations of follow-up for CsAresistant and nontreated patients were 41 (23-92) and 48 (28-106) months, respectively. Twenty patients (36\%) were responsive to CSA and were followed up for a median (IQR) of 81 (47-115) months. Progression to ESRF occurred in $10 \%$ of CsA-responsive patients versus $60 \%$ of CsA-resistant patients and $62 \%$ of nontreated patients $(P=0.002)$. No sign of renal fibrosis related to drug toxicity was observed in renal biopsies performed at 5-year intervals.
\end{abstract}

Conclusions: This retrospective analysis of SRNS documented a persistent antiproteinuric effect of long-term CsA ( $>2$ years) in the absence of renal fibrosis. Although sensitivity to CsA was associated with normal renal function, resistance or intolerance was associated with progression to ESRF. These data suggest that CsA may have a role in the treatment of patients with SRNS. (Clin Ther. 2004;26:1411-1418) Copyright (C) 2004 Excerpta Medica, Inc.

Key words: nephrotic syndrome, focal segmental glomerulosclerosis, cyclosporine, cyclophosphamide, endstage renal failure. 


\section{INTRODUCTION}

Steroid-resistant nephrotic syndrome (SRNS) associated with focal segmental glomerulosclerosis (FSGS) is one of the most frequent glomerular diseases in children and adults ${ }^{1}$ and has emerged as a leading cause of end-stage renal failure (ESRF). ${ }^{1}$ The estimated incidence in US children (age, $<14$ years) is 2.0 to 2.7 per 100,000 per year, with a cumulative prevalence of 16 per $100,000 .^{2}$ Geographic or ethnic differences have been reported, with a 6 -fold greater incidence in Asian than in European children. ${ }^{2,3}$ SRNS appears to be a clinical heterogeneous condition characterized by histologic variants, ${ }^{4-6}$ different genetic backgrounds, ${ }^{7-9}$ and variable responses to drugs. ${ }^{10,11}$ The pathologic picture in these patients is mainly represented by focal area of segmental glomerulosclerosis that often coexists with diffuse mesangial immunoglobulin (Ig) $\mathrm{M}$ deposition, ${ }^{12,13}$ and the boundary between the 2 entities cannot always be defined. ${ }^{14,15}$ The typical clinical hallmark of these patients is strict resistance to steroids that are invariably used as a first-line therapy.

Cyclosporine (CSA) was introduced in 1993 as a therapeutic option for treating patients with SRNS ${ }^{16}$ because it had been proved effective in the short-term (ie, within 1 year) management of proteinuria, ${ }^{17,18}$ and randomized studies ${ }^{17-19}$ confirm this effect. Unfortunately, the long-term administration of CsA (over years) carries the potential for renal toxicity, ${ }^{20}$ and historical trials considered only short-term regimens lasting $\leq 1$ year. Now, long-term ( $>4$ years) regimens with low-dose (2-3 mg/kg body weight) CsA are widely applied in clinical practice, thus allowing satisfactory control of proteinuria and potentially low toxicity. Studies on the effectiveness and safety of CSA in SRNS are needed to consolidate the wide clinical popularity of the drug. Despite clear limitations, retrospective, nonrandomized studies should represent a first-line approach to defining the clinical outcome in patients with SRNS treated for $>2$ years with CsA. A central issue in such studies is to exclude carriers of inherited SRNS due to mutations of one of the proteins of the slit-diaphragm because they mimic noninherited types while presenting invariable and rapid progression to ESRF. In particular, mutations of podocin, one of the major genes causing a recessive type of FSGS, have been described in a significant proportion $(\sim 10 \%)$ of patients with sporadic
SRNS who inherited the defects as an autosomal recessive trait ${ }^{21-23}$; these patients should be identified and excluded in studies looking at the pharmacologic approach to SRNS.

In the current study, we considered the clinical outcome of patients with SRNS who had been treated with CsA for $>2$ years. The primary objective was to evaluate renal function after years of treatment compared with that of nontreated or CsA-resistant patients. A secondary objective was to identify any renal effects related to the use of CsA, with a major emphasis on renal fibrosis (a major renal adverse effect of the drug). ${ }^{20}$

\section{PATIENTS AND METHODS}

We retrospectively studied patients of all ages with sporadic SRNS who had been followed up for >2 years (between 1970 and 2002) at 4 Italian clinical institutions and for whom DNA was collected for molecular analysis. Inclusion criteria were the presence of nephrotic proteinuria (urinary protein excretion, $>40 \mathrm{mg} / \mathrm{kg} \cdot \mathrm{d}$ ) and the availability of a renal biopsy with proven FSGS lesions ( $\geq 1$ segmental sclerotic area) with or without IgM deposition. In all cases, the therapeutic approach was to start with steroids adhering to widely used regimens ${ }^{10,11}\left(60 \mathrm{mg} / \mathrm{m}^{2} \cdot \mathrm{d}\right.$ for $30-60$ days $)$ and, in cases of nonresponsiveness (partial or global), steroids were combined or substituted with cyclophosphamide (2 $\mathrm{mg} / \mathrm{kg} \cdot \mathrm{d}$ for 60 days) and/or with CsA ( $5 \mathrm{mg} / \mathrm{kg} \cdot \mathrm{d}$ starting dose, followed by tapering to reach the minimum dose required for maintaining CsA serum trough levels between 50 and $100 \mathrm{ng} / \mathrm{mL}$ ). In cases of persistent steroid/CsA resistance, methylprednisolone was given in pulses (10 $\mathrm{mg} / \mathrm{kg} ; 6$ cycles). Steroid resistance was considered to be the failure to achieve remission of proteinuria (urinary protein excretion, $<2 \mathrm{mg} / \mathrm{kg} \cdot \mathrm{d}$ ) after 4 weeks of steroid treatment. CsA resistance was the failure to modify proteinuria after 8 weeks of CsA at a starting dose of $5 \mathrm{mg} / \mathrm{kg}$. CsA intolerance was the worsening of renal function (creatinine clearance $<70 \%$ of pre-CsA values) and/or the increment of mean arterial blood pressure $(\geq 50 \%)$ after the start of therapy.

General clinical parameters studied included blood cell counts; levels of serum creatinine, urea cholesterol, and triglycerides; and liver function tests performed according to international standardized procedures. Renal biopsies were processed using standard procedures that included hematoxylin-eosin, periodic 
acid-Schiff, and green Masson stainings of formalinincluded pieces; immunofluorescence of frozen samples was carried out with a panel of antiserum protein antibodies against $\operatorname{IgA}, \operatorname{IgM}, \operatorname{IgG}, \mathrm{C} 3$, and C4. Biopsy samples were evaluated by a pathologist, who discussed all pertinent aspects with the referring clinicians. The relevant clinical and pathologic features (ie, sex, age at onset of proteinuria, and progression toward ESRF) are reported. The basic molecular approach included sequencing of podocin, nephrin, and $\alpha$ actinin 4 , which are proteins of the glomerular slitdiaphragm whose mutations are invariably associated with SRNS and rapid progression to ESRF. Adult patients or parents of patients aged $<18$ years provided written informed consent for DNA analysis and for the statistical review of the clinical variables. Consensual patients were numbered, and data were evaluated on an anonymous basis, access key numbers being in possession of the senior author (G.M.G.). We also had permission to collect blood and analyze clinical data from patients with SRNS starting from 1995 by the chief of the Department of Nephrology at G. Gaslini Children's Hospital; this permission was renewed in 2001. At the time this study was initiated, this approval was required for retrospective studies.

\section{Mutation Analysis}

We obtained peripheral blood samples for genetic analysis from the enrolled patients and from selected parents and siblings. Five milliliters of blood in ethylenediaminetetraacetic acid (EDTA) was obtained on the morning after an overnight fast, rapidly frozen in plastic vials, and maintained at $-20^{\circ} \mathrm{C}$. Genomic DNA was extracted according to standard procedures. Molecular analyses of podocin and nephrin were performed by direct sequencing as described previously.,9,21 Primer sequences for podocin were selected on the basis of literature reports by different groups. ${ }^{7}$ In particular, for exons 2 and 6 primer design, we followed the guidelines of Ruf et $\mathrm{al}^{23}$ to avoid the presence of a recognized single nucleotide polymorphism. Exons were amplified by polymerase chain reaction using flanking intronic primers and subjected to automatic sequence analysis by dye-terminator reaction (ABI 3100 Automated Sequencer, Applera Corporation, Milan, Italy). For $\alpha$ actinin 4, the "hot-spot" A682G and C695T mutations at exon 8 were detected by restriction site analyses as described by Kaplan et al. ${ }^{8}$

\section{Statistical Analysis}

Data are expressed as median (interquartile range $[\mathrm{IQR}]$ ). Because all quantitative variables did not present a normal distribution, box plots and nonparametric tests were used to represent and compare the distributions of age at onset of proteinuria in groups defined by the response to pharmacologic treatments. In box plots, horizontal lines display the 10th, 25th, 50th, $75 \mathrm{th}$, and 90 th percentiles of a variable.

The influence of any general (eg, sex, age at onset of proteinuria) and clinical (eg, histology, pharmacologic treatments) variables on the progression to ESRF was investigated using the multivariate Cox regression model (proportional hazards).

Kruskal-Wallis analysis was employed to compare the median age of patients responsive to CsA with that of those who were resistant or nontreated. Kaplan-Meier plots and log-rank tests were used to assess whether survival differed significantly among subgroups defined according to sex or pharmacologic treatment. $P$ values $<0.05$ were considered significant. StatView version 5.0.1 (SAS Institute Inc., Cary, North Carolina) was used in the statistical approach. In this analysis, the event time variable measures the time elapsed between onset of proteinuria and the beginning of dialysis. Because the observational period ended in December 2002, those patients who did not reach the event at that time were censored.

\section{RESULTS}

The cohort of 157 patients described here was part of a large series of patients with SRNS and FSGS who had been followed up between 1970 and 2002 at 4 Italian institutions and for whom clinical data of the follow-up were available. This series does not include all patients with SRNS followed up at these institutions, but only those for whom a DNA sample was collected, allowing for a molecular classification. Mutation screenings for components of the slit-diaphragm were, in fact, considered essential to exclude those patients who presented with an inherited type of FSGS, still having sporadic disease. Mutations of podocin were found in 17 patients $(11 \%)$; nephrin was mutated in $1(<1 \%)$. According to our protocol, carriers of podocin and nephrin mutations were excluded from the present study and are described in detail elsewhere ${ }^{21,24}$; they represent a separate cohort of patients with 
invariable progression to ESRF despite any pharmacologic treatment.

Of the remaining 139 patients (84 men, 55 women; median [IQR] age at onset of proteinuria, 12 [4-32] years), 45 (32\%) were treated with cyclophosphamide without any clinically relevant effect (Table). Eighty-four patients (60\%) received no treatment, and $55(40 \%)$ were treated with CsA. Of these 55 patients, 35 (64\%) were found to be resistant or intolerant to the drug; the drug was then withdrawn in these patients. In 20 patients $(36 \%)$, CsA induced a complete remission of proteinuria, which is stable normalization of urinary protein levels over time (median [IQR] follow-up, 81 [47-115] months). The median (IQR) follow-up for patients sensitive to CsA was 81 (47-115) months $(50 \%$ of cases had been followed up for 4-10 years). Patients who were CsA resistant or were nontreated had shorter median (IQR) followups of 41 (23-92) and 48 (28-106) months, respectively, as most of these patients progressed to ESRF during the follow-up period. No cases of liver damage and/or gastrointestinal disturbances (eg, nausea, vomiting, abdominal pain, anemia, hyperkalemia) were observed.

As shown in Figure 1, CsA was used mostly in young patients with steroid resistance. In fact, most patients who had been treated with CsA were children (median age, 5 and 8 years for responders and resistant patients, respectively; IQR for responders and resistant patients combined, 8-10); the median (IQR) age of nontreated patients was 19 years (8-44 $[\mathrm{H}=17.998 ; P<0.001)$. CsA sensitivity was associated with long-term (over years) preservation of renal function. No single general or clinical parameter was predictive of the antiproteinuric response of CsA. In the panel of clinical factors associated with CsA response and outcome of renal function, we did not consider prevalence of hypertension and treatment because the pharmacologic approach to hypertension varied over years, and clear information was not available. Figure 2 shows the cumulative survival (considering ESRF that required a substitutive treatment as the end point) of responders and CsA-resistant patients. Two of 20 responders (10\%) had progression to ESRF compared with 21 of $35(60 \%)$ in the

Table. Baseline demographic and clinical characteristics of patients with steroid-resistant nephrotic syndrome followed up for $>2$ years at 4 Italian institutions between 1970 and $2002 .^{* \dagger}$

\begin{tabular}{|c|c|c|c|c|}
\hline Characteristic & $\begin{array}{l}\text { CsA Responsive } \\
(n=20)\end{array}$ & $\begin{array}{l}\text { CsA Intolerant or Resistant } \\
\qquad(n=35)\end{array}$ & $\begin{array}{l}\text { No CsA Treatment } \\
(n=84)\end{array}$ & $\begin{array}{l}\text { All Patients } \\
(\mathrm{N}=139)\end{array}$ \\
\hline \multicolumn{5}{|l|}{ Demographic } \\
\hline \multicolumn{5}{|l|}{ Sex, no. (\%) } \\
\hline Male & $14(70)$ & $22(63)$ & $48(57)$ & $84(60)$ \\
\hline Female & $6(30)$ & $13(37)$ & $36(43)$ & $55(40)$ \\
\hline \multicolumn{5}{|l|}{ Clinical } \\
\hline $\begin{array}{l}\text { Age at proteinuria onset, median (IQR), y } \\
\text { Histology, no. (\%) }\end{array}$ & $5(3-11)$ & $8(4-14)$ & $19(8-44)$ & $12(4-32)$ \\
\hline FSGS & $17(85)$ & $28(80)$ & $78(93)$ & $123(88)$ \\
\hline Mes.-lgM & $3(15)$ & $7(20)$ & $6(7)$ & $16(12)$ \\
\hline \multicolumn{5}{|l|}{ Cyclophosphamide response, no. (\%) } \\
\hline Responsive & $0(0)$ & I (3) & $5(6)$ & $6(4)$ \\
\hline Unresponsive & $9(45)$ & $14(40)$ & $16(19)$ & $39(28)$ \\
\hline Not determined & II (55) & $20(57)$ & $63(75)$ & $94(68)$ \\
\hline ESRF, no. (\%) & $2(10)$ & $21(60)$ & $52(62)$ & $75(54)$ \\
\hline Follow-up, median (IQR), mo & $81(47-115)$ & $41(23-92)$ & $48(28-106)$ & $48(28-105)$ \\
\hline
\end{tabular}

$\mathrm{CSA}=$ cyclosporine; $\mathrm{IQR}=$ interquartile range; $\mathrm{FSGS}=$ focal segmental glomerulosclerosis; Mes. IgM = mesangial proliferative glomerulonephritis with immunoglobulin $M$ deposition; ESRF = end-stage renal failure.

"No significant between-group differences were found.

tThis cohort was part of a large series of nephrotic patients for whom a DNA sample was available and was utilized for screening of podocin and nephrin mutations. Those patients carrying a mutation were excluded from the study and described elsewhere. ${ }^{21.24}$ 


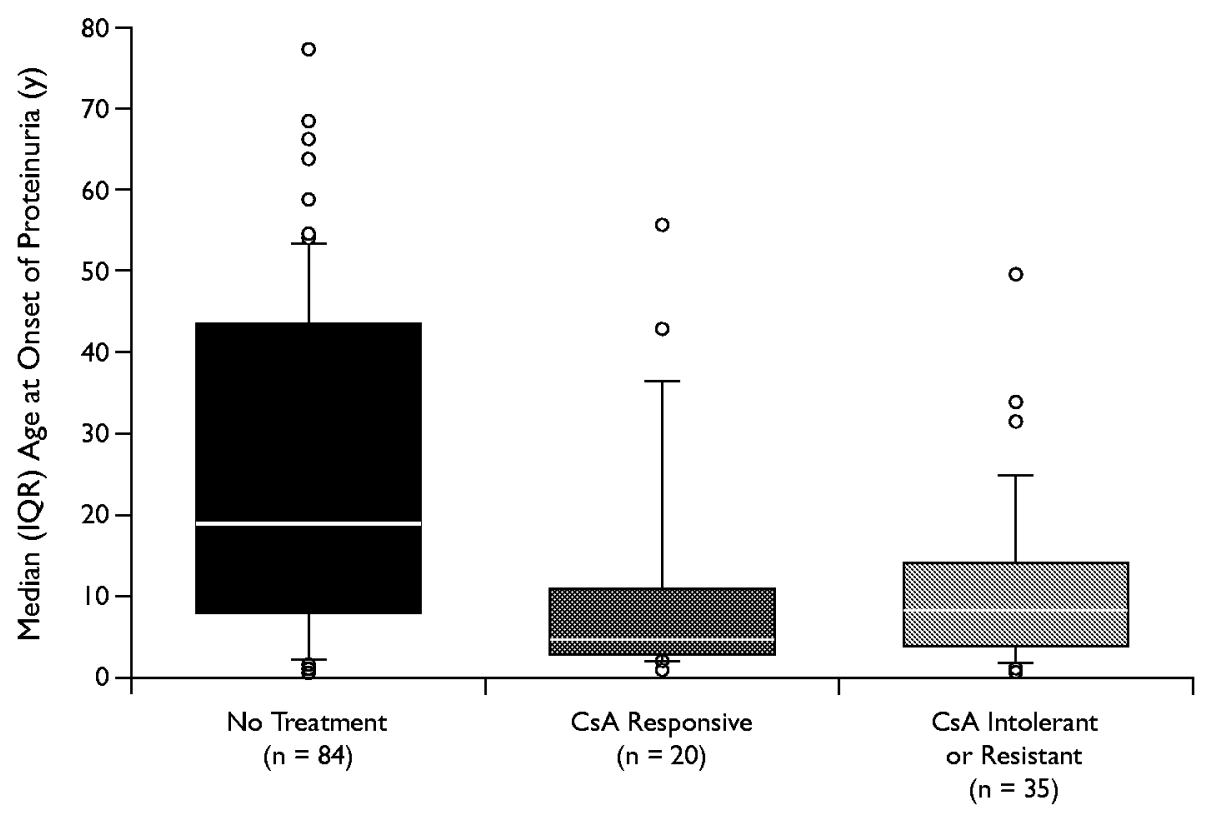

Figure I. Median age at onset of proteinuria in patients with steroid-resistant nephrotic syndrome. Because all quantitative variables did not present a normal distribution, box plots and nonparametric tests were used to represent and compare the distributions of age at onset of proteinuria in groups defined by the response to pharmacologic treatments. In box plots, horizontal lines display the 10th, 25th, 50th, 75th, and 90th percentiles of a variable. Patients who were treated with cyclosporine (CsA) were younger than those who were not treated $(\mathrm{H}=17.998$; $P<0.00$ I [Kruskal-Wallis]).

resistant group and 52 of 84 nontreated patients $(62 \%)(P=0.002)$. Thirteen patients $(60 \%)$ in the group with CsA sensitivity presented with $\geq 1$ proteinuria relapse after a follow-up of $>3$ years and were treated with steroids. In a few cases, low-dose steroids $(0.1-0.5 \mathrm{mg} / \mathrm{kg} \cdot \mathrm{d})$ were necessary to maintain proteinuria within the normal range, suggesting that a type of steroid dependence may occur in some patients, in which case CsA was able to determine a change in sensitivity to steroids from strict resistance to dependence. Patients treated with CsA for $>4$ years had a renal biopsy at 5-year intervals, and in no instance did we observe relevant tubulointerstitial fibrosis. Accordingly, the drug was not withdrawn in any patient.

\section{DISCUSSION}

SRNS associated with FSGS is an emerging cause of ESRF in children and adults. ${ }^{1,25}$ Because FSGS, and SRNS in general, is not a homogeneous disease but has different clinical and genetic backgrounds, factors associated with progression must be identified. The first issue is the genetic background relative to proteins of the slit-diaphragm. ${ }^{7-9}$ Some reports ${ }^{21,23,24,26}$ published between 2001 and 2004 demonstrated that a significant proportion of children with SRNS and FSGS have homozygous mutations of podocin. In most of these patients, whose disease was not distinguishable on clinical grounds from idiopathic FSGS, the disease progressed to ESRF within the second decade of life, indicating the key involvement genetic background has in progression. A second potential factor associated with progression to ESRF in patients with FSGS is responsiveness to therapies; steroid sensitivity has been indicated previously as the most useful criterion to identify patients whose disease progresses. ${ }^{27,28} \mathrm{~A}$ basic issue is how to define steroid resistance because new reports suggest an IV route of methylprednisolone pulses, ${ }^{29}$ and data from the literature clearly show that it is in patients who are resistant to steroids that progression is common. ${ }^{30,31}$ In steroid-resistant patients, CsA represents 
the second line of defense. This drug was introduced in 1993 as a therapeutic option for treating SRNS, but its use was restricted to short periods. ${ }^{16}$ One major reason for brief courses of treatment with CsA is the strong profibrotic effect of the drug that has been documented. ${ }^{20}$ Currently, CsA is used at a low dose (2-3 mg/kg body weight) for $\geq 5$ years. ${ }^{16,30}$ Few longterm studies considering the adverse effects of this low dose are available. A literature search of PubMed for articles on CsA therapy for SRNS published between 1987 and 2004 (key words: nephrotic syndrome and cyclosporine with restriction to focal segmental glomerulosclerosis) identified 47 articles, most referring to drug treatment of 1 year. Our study cohort consisting of patients with sensitivity to CsA had a median 81-month follow-up and represents the group with idiopathic SRNS with the longest period so far reported.
With the clear limitations of a retrospective review of nonrandomized patients, the results of our study report data on a cohort of patients with FSGS characterized for molecular aspects of the slit-diaphragm proteins who had been treated for significant periods of time. Patients with steroid and CsA resistance represent the bulk of our study cohort, and data here confirm that progression to ESRF is frequent in these cases and involved $~ 50 \%$ of patients. According to the cumulative survival rate reported in Figure 2, sensitivity to CsA appears to be the major factor influencing long-term outcome in patients with SRNS. This behavior was mainly influenced by those patients who had remission of proteinuria during pharmacologic treatment, evidenced by the fact that only 2 of the cohort of 20 patients in whom CsA elicited an antiproteinuric effect had progression to ESRF. What mediates progression to ESRF in patients intolerant to CsA is currently

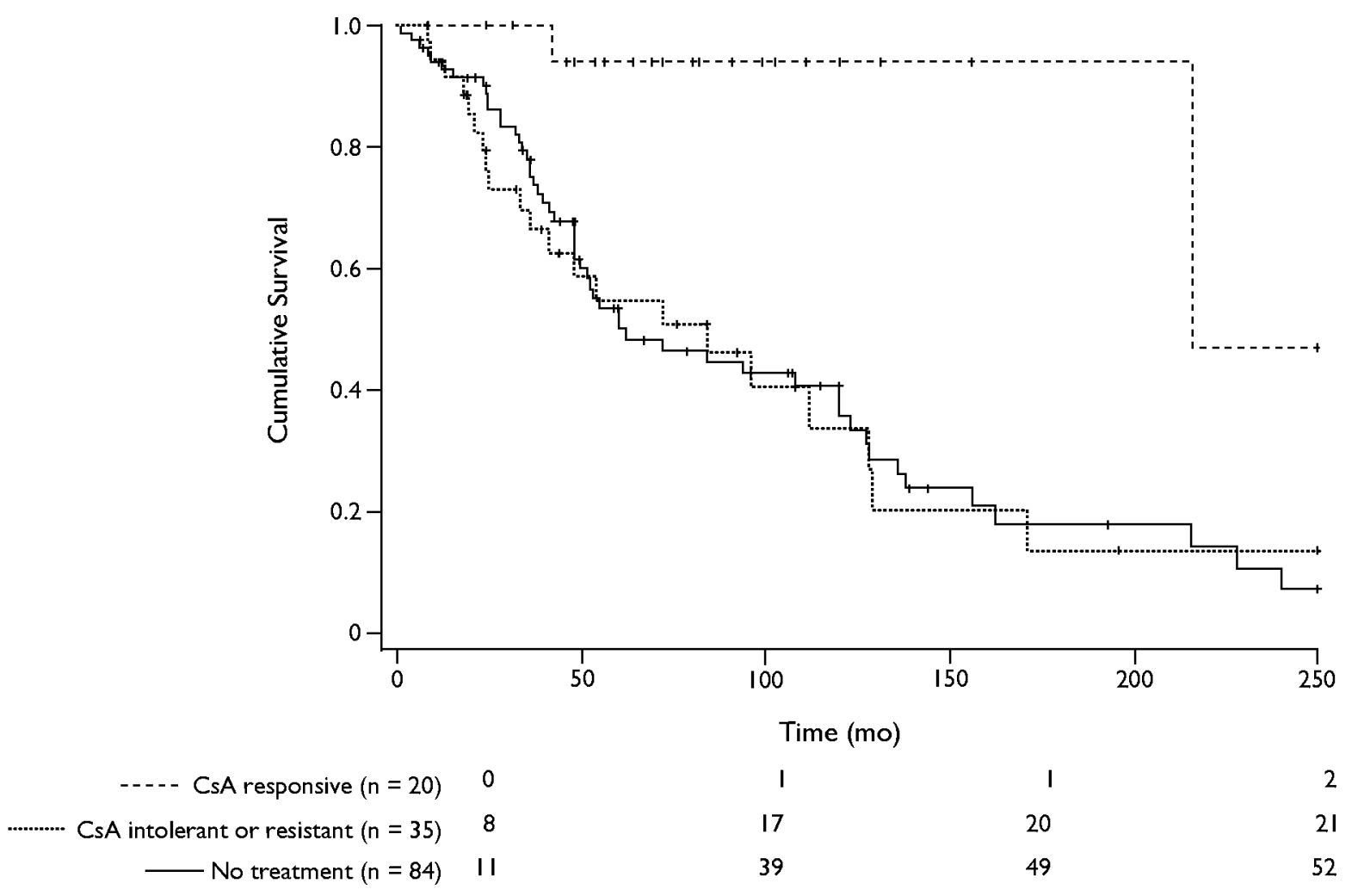

Figure 2. Cumulative survival in patients with steroid-resistant nephrotic syndrome who were treated $(n=55)$ or not treated ( $n=84$ ) with cyclosporine (CsA). The treatment group was subdivided into patients who had an antiproteinuric response to $\operatorname{CsA}(n=20)$ and patients who were intolerant or had no response at all $(n=35)$. The end point was end-stage renal failure that required a substitutive treatment. Events are indicated at the bottom. Censored patients are indicated as a vertical tract in each cumulative curve. Log-rank test, chi-square test, $12.448 ; d f=2 ; P=0.002$. 
unknown. It is reasonable to hypothesize that intractable proteinuria triggers a series of events leading to tubulointerstitial fibrosis. However, evaluation of these patients was not within the scope of the current study, and studies on genetic predisposition for developing fibrosis are in progress. The observation that CsA may reduce proteinuria in some patients who were resistant to steroids is not new and confirms previous data by Cattran et $\mathrm{al}^{19}$ and Niaudet. ${ }^{31}$ Our report incorporates the observation that CsA not only improves proteinuria but also ameliorates the long-term outcome and reduces the incidence of ESRF in patients with SRNS. Data from the current study demonstrate that CsA at a low dose may be used for several years without clinically relevant adverse effects.

Unfortunately, the present study indicates that CsA is used mainly in children, which leads to the question of why most adults with SRNS are not treated with this drug (median age of nontreated patients, 18 years). Overall, data on long-term CsA treatment are encouraging because most patients belonging to both categories were still being treated after $>4$ years and had normal renal function and unremarkable renal histologic findings.

\section{CONCLUSIONS}

In this study of patients with SRNS and FSGS, low-dose CSA reduced proteinuria in some patients with SRNS. This beneficial effect was associated with maintenance of normal renal function over time. Renal fibrosis was rare in patients treated with CsA for $>4$ years, suggesting a good safety profile of the drug with these patients. These data suggest that low-dose CSA may have a role in the long-term management of SRNS.

\section{ACKNOWLEDGMENTS}

We are indebted to Prof. Laura A. Zonta for statistical overview and Anna Capurro for her invaluable help in the preparation of this manuscript.

This study was supported by a grant from the Italian Ministry of Health ("Pathogenesis of diseases of the renal filtration barrier," Contract no. 178/02) and from the G. Gaslini Institute (Ricerca Corrente).

\section{REFERENCES}

1. Braden GL, Mulhern JG, O'Shea MH, et al. Changing incidence of glomerular diseases in adults. Am J Kidney Dis. 2000;35:878-883.
2. McEnery PT, Strife CF. Nephrotic syndrome in childhood. Management and treatment in patients with minimal change disease, mesangial proliferation, or focal glomerulosclerosis. Pediatr Clin North Am. 1982;29:875894.

3. Sharples PM, Poulton J, White RH. Steroid responsive nephrotic syndrome is more common in Asians. Arch Dis Child. 1985;60:1014-1017.

4. Border WA. Distinguishing minimal-change disease from mesangial disorders. Kidney Int. 1988;34:419434.

5. Korbet SM. Primary focal segmental glomerulosclerosis. J Am Soc Nephrol. 1998;9:1333-1340.

6. Myllymaki J, Saha H, Mustonen J, et al. IgM nephropathy: Clinical picture and long-term prognosis. Am J Kidney Dis. 2003:41:343-350

7. Boute N, Gribouval O, Roselli S, et al. NPHS2, encoding the glomerular protein podocin, is mutated in autosomal recessive steroid-resistant nephrotic syndrome [published correction appears in Nat Genet. 2000;25:125]. Nat Genet. 2000;24:349-354

8. Kaplan JM, Kim SH, North KN, et al. Mutations in ACTN4, encoding alpha-actinin-4, cause familial focal segmental glomerulosclerosis. Nat Genet. 2000;24:251256.

9. Lenkkeri U, Mannikko M, McCready P, et al. Structure of the gene for congenital nephrotic syndrome of the Finnish type (NPHS1) and characterization of mutations. Am J Hum Genet. 1999;64:51-61.

10. Primary nephrotic syndrome in children: Clinical significance of histopathologic variants of minimal change and of diffuse mesangial hypercellularity. A Report of the International Study of Kidney Disease in Children. Kidney Int. 1981;20:765-771.

11. Prospective, controlled trial of cyclophosphamide therapy in children with nephrotic syndrome. Report of the International Study of Kidney Disease in Children. Lancet. 1974;2:423-427.

12. Bonilla-Felix M, Parra C, Dajani T, et al. Changing patterns in the histopathology of idiopathic nephrotic syndrome in children. Kidney Int. 1999;55:1885-1890.

13. Korbet SM, Schwartz MM, Lewis EJ. Primary focal segmental glomerulosclerosis: Clinical course and response to therapy. Am J Kidney Dis. 1994;23:773783.

14. Gonzalo A, Mampaso F, Gallego N, et al. Clinical significance of $\operatorname{IgM}$ mesangial deposits in the nephrotic syndrome. Nephron. 1985;41:246-249. 
15. McAdams AJ, Valentini RP, Welch TR. The nonspecificity of focal segmental glomerulosclerosis. The defining characteristics of primary focal glomerulosclerosis, mesangial proliferation, and minimal change. Medicine (Baltimore). 1997;76:42-52.

16. Ponticelli C, Rizzoni G, Edefonti A, et al. A randomized trial of cyclosporine in steroid-resistant idiopathic nephrotic syndrome. Kidney Int. 1993;43:1377-1384.

17. Hymes LC. Steroid-resistant, cyclosporine-responsive, relapsing nephrotic syndrome. Pediatr Nephrol. 1995; 9:137-139

18. Ingulli E, Singh A, Baqi N, et al. Aggressive, long-term cyclosporine therapy for steroid-resistant focal segmental glomerulosclerosis. J Am Soc Nephrol. 1995;5: 1820-1825.

19. Cattran DC, Appel GB, Hebert LA, et al, for the North America Nephrotic Syndrome Study Group. A randomized trial of cyclosporine in patients with steroidresistant focal segmental glomerulosclerosis. Kidney Int. 1999;56:2220-2226.

20. Ghiggeri GM, Altieri P, Oleggini R, et al. Cyclosporine enhances the synthesis of selected extracellular matrix proteins by renal cells "in culture". Different cell responses and phenotype characterization. Transplantation. 1994; 57:1382-1388.

21. Caridi G, Bertelli R, Carrea A, et al. Prevalence, genetics, and clinical features of patients carrying podocin mutations in steroid-resistant nonfamilial focal segmental glomerulosclerosis. J Am Soc Nephrol. 2001; $12: 2742-2746$.

22. Karle SM, Uetz B, Ronner V, et al. Novel mutations in NPHS2 detected in both familial and sporadic steroidresistant nephrotic syndrome. J Am Soc Nephrol. 2002; 13:388-393.

23. Ruf RG, Lichtenberger A, Karle SM, et al, for the Arbeitsgemeinschaft fur Padiatrische Nephrologie
Study Group. Patients with mutations in NPHS2 (podocin) do not respond to standard steroid treatment of nephrotic syndrome. J Am Soc Nephrol. 2004; 15:722-732.

24. Caridi G, Bertelli R, Scolari F, et al. Podocin mutations in sporadic focal-segmental glomerulosclerosis occurring in adulthood. Kidney Int. 2003;64:365.

25. Schwartz MM, Korbet SM, Rydell J, et al. Primary focal segmental glomerular sclerosis in adults: Prognostic value of histologic variants. Am J Kidney Dis. 1995; 25:845-852.

26. Caridi G, Bertelli R, Di Duca M, et al. Broadening the spectrum of diseases related to podocin mutations. J Am Soc Nephrol. 2003;14:1278-1286.

27. Berns JS, Gaudio KM, Krassner LS, et al. Steroidresponsive nephrotic syndrome of childhood: A longterm study of clinical course, histopathology, efficacy of cyclophosphamide therapy, and effects on growth. Am J Kidney Dis. 1987;9:108-114.

28. Trompeter RS, Lloyd BW, Hicks J, et al. Long-term outcome for children with minimal-change nephrotic syndrome. Lancet. 1985;1:368-370.

29. Hari P, Bagga A, Jindal N, Srivastava RN. Treatment of focal glomerulosclerosis with pulse steroids and oral cyclophosphamide. Pediatr Nephrol. 2001;16:901905.

30. Meyrier A, Noel LH, Auriche P, Callard P, for the Collaborative Group of the Societe de Nephrologie. Long-term renal tolerance of cyclosporin A treatment in adult idiopathic nephrotic syndrome. Kidney Int 1994:45:1446-1456.

31. Niaudet P, for the French Society of Pediatric Nephrology. Treatment of childhood steroid-resistant idiopathic nephrosis with a combination of cyclosporine and prednisone. J Pediatr. 1994;125:981986.

Address correspondence to: Gian Marco Ghiggeri, MD, Department of Nephrology, G. Gaslini Children's Hospital of Genoa, Largo Gaslini 5, 16148 Genova, Italy. E-mail: labnefro@ospedale-gaslini.ge.it 